\title{
Advances in Single Crystal Growth and Annealing Treatment of Electron-doped HTSC
}

\author{
Michael Lambacher, Toni Helm, Mark Kartsovnik, and Andreas Erb \\ Walther-Meißner-Institut, Bayerische Akademie der Wissenschaften, \\ Walther-Meißner-Straße 8, D-85748 Garching, Germany.
}

\begin{abstract}
High quality electron-doped HTSC single crystals of $\mathrm{Pr}_{2-\mathrm{x}} \mathrm{Ce}_{\mathrm{x}} \mathrm{CuO}_{4+\delta}$ and $\mathrm{Nd}_{2-\mathrm{x}} \mathrm{Ce}_{\mathrm{x}} \mathrm{CuO}_{4+\delta}$ have been successfully grown by the container-free traveling solvent floating zone technique. The optimally doped $\mathrm{Pr}_{2-\mathrm{x}} \mathrm{Ce}_{\mathrm{x}} \mathrm{CuO}_{4+\delta}$ and $\mathrm{Nd}_{2-\mathrm{x}} \mathrm{Ce}_{\mathrm{x}} \mathrm{CuO}_{4+\delta}$ crystals have transition temperatures $T_{\mathrm{c}}$ of $25 \mathrm{~K}$ and $23.5 \mathrm{~K}$, respectively, with a transition width of less than $1 \mathrm{~K}$. We found a strong dependence of the optimal growth parameters on the Ce content $x$. We discuss the optimization of the post-growth annealing treatment of the samples, the doping extension of the superconducting dome for both compounds as well as the role of excess oxygen. The absolute oxygen content of the as-grown crystals is determined from thermogravimetric experiments and is found to be $\geq 4.0$. This oxygen surplus is nearly completely removed by a post-growth annealing treatment. The reduction process is reversible as demonstrated by magnetization measurements. In as-grown samples the excess oxygen resides on the apical site $\mathrm{O}(3)$. This apical oxygen has nearly no doping effect, but rather influences the evolution of superconductivity by inducing additional disorder in the $\mathrm{CuO}_{2}$ layers. The very high crystal quality of $\mathrm{Nd}_{2-\mathrm{x}} \mathrm{Ce}_{\mathrm{x}} \mathrm{CuO}_{4+\delta}$ is particularly manifest in magnetic quantum oscillations observed on several samples at different doping levels. They provide a unique opportunity of studying the Fermi surface and its dependence on the carrier concentration in the bulk of the crystals.
\end{abstract}




\section{INTRODUCTION}

In comparison to the various families of the hole-doped cuprate superconductors the electron-doped 214 compounds $\mathrm{Ln}_{2-x} \mathrm{Ce}_{\mathrm{x}} \mathrm{CuO}_{4+\delta}$ (with $\mathrm{Ln}=\mathrm{Pr}, \mathrm{Nd}, \mathrm{Sm}, \mathrm{Eu}, \ldots$ ) have been studied less intensively, even though this system is known for more than 15 years [1]. There are different reasons for this. First, the growth of high quality single crystals and the postgrowth annealing treatment are very demanding. Second, the controlled and reproducible preparation of well characterized samples for different experiments, such as well-oriented samples with clean surfaces for optical spectroscopies, is difficult. Despite these drawbacks, the electron-doped 214-compounds are very attractive as a sample set for systematic investigations for two reasons: (i) They are solid solutions with a simple tetragonal crystal structure and nearly the whole phase diagram can be probed using only a single compound. Starting from the undoped antiferromagnetic insulator $\mathrm{Ln}_{2} \mathrm{CuO}_{4+\delta}$ (with $\mathrm{Ln}=\mathrm{Pr}, \mathrm{Nd}, \mathrm{Sm}$, $\mathrm{Eu}, . .$.$) , samples with different electronic ground states up to the overdoped metallic regime$ can be grown by gradually doping with tetravalent Ce. (ii) With $\mathrm{La}_{2-\mathrm{x}} \mathrm{Sr}_{\mathrm{x}} \mathrm{CuO}_{4-\delta}$ there is a corresponding hole-doped compound with a similar crystal structure and critical temperature $T_{\mathrm{c}}$. This provides the unique opportunity to analyze similarities and differences in the phase diagram of the electron- and hole-doped compounds.

\section{GROWTH PROCESS AND GROWTH PARAMETERS}

\section{A. Crystal Growth}

The growth of high quality 214-crystals in crucibles from a CuO-rich melt is difficult for various reasons: Firstly, deviations of the distribution coefficient from unity in these systems give rise to gradients in the dopant concentration of the grown crystals. Secondly, it is well

known from other cuprate systems, such as $\mathrm{YBa}_{2} \mathrm{Cu}_{3} \mathrm{O}_{7-\delta}$ [2], as well as from previous work on $\mathrm{Nd}_{2-\mathrm{x}} \mathrm{Ce}_{\mathrm{x}} \mathrm{CuO}_{4+\delta}$ [3], that the aggressive melts partly dissolve the most common available crucible materials. This unwanted corrosion leads to impurities and, in turn, to a deterioration of the crystal quality. We also note that the 214-compounds are incongruently melting solid solutions [4]. Therefore, the growth process is restricted to a small interval between the peritectic and eutectic points of the compositional phase diagram.

The Traveling Solvent Floating Zone (TSFZ) technique provides the opportunity and 
flexibility to work at a certain favorable working point within this small growth window by using a small $\mathrm{CuO}$-rich flux pellet and a corresponding atmosphere and temperature. The flux pellet forms a vertical local melt, held by surface tension, where the polycrystalline feed material is dissolved at the top and re-crystallized at the bottom on a seed. Using suitable growth conditions, an equilibrium between growth and solubility rate can be obtained. In this way large crystals of several centimeters length can be grown under accurately controllable stable conditions (flux composition, temperature, oxygen partial pressure). This is the basic prerequisite for homogeneous crystals.

For the growth of a series of $\mathrm{Ln}_{2-x} \mathrm{Ce}_{\mathrm{x}} \mathrm{CuO}_{4+\delta}$ crystals (with $\mathrm{Ln}=\mathrm{Nd}$, Pr) we have used a 4-mirror furnace with four $300 \mathrm{~W}$ halogen lamps. The polycrystalline feed rods and flux pellets were prepared in the following way: For the feed rods the corresponding rare earth oxides and $\mathrm{CuO}$ (with purity of 99.99\%) were mixed according to the desired stoichiometric composition of the 214-compounds. Then, phase pure ceramic samples were obtained by a five-fold pre-reaction of the powders at temperatures of $900^{\circ} \mathrm{C}, 920^{\circ} \mathrm{C}, 950^{\circ} \mathrm{C}$, and two times at $980^{\circ} \mathrm{C}$ for $10 \mathrm{~h}$ in air. Between each calcination step the pre-reacted powder was homogenized by grinding. The multiple calcination promotes the homogeneity. The phase formation of the powder was checked by X-ray powder diffraction. The phase pure ceramic samples were pressed hydrostatically to rods of $7 \mathrm{~mm}$ in diameter and $140 \mathrm{~mm}$ in length. These polycrystalline rods were sintered in air at temperatures of $1050^{\circ} \mathrm{C}, 1100^{\circ} \mathrm{C}$, and $1200^{\circ} \mathrm{C}$ for $5 \mathrm{~h}$, respectively. These high temperatures close to the peritectic temperature are important in order to increase the density, avoiding the absorption of the liquid flux during crystal growth. The flux material with the composition ratio $\left[(2-x) \cdot 1 / 2 \cdot\left(\operatorname{Ln}_{2} \mathrm{O}_{3}\right)+\right.$ $\left.x \cdot \mathrm{CeO}_{2}\right] / \mathrm{CuO}=15 / 85$ was pre-reacted in the same way as the feed rods, followed by an annealing step at $1010^{\circ} \mathrm{C}$ for $10 \mathrm{~h}$ in air.

For single crystal growth, a piece of a polycrystalline rod was used as a seed. On this seed a flux pellet of $0.35-0.40 \mathrm{~g}$ was placed. The mass and size of this flux pellet needs to be adjusted to the dimensions of the radiation focus and the diameter of the rods. The vertical molten zone has a diameter and length of $5-6 \mathrm{~mm}$ and can easily be held by surface tension during the entire growth process. The growth atmosphere, i.e. the oxygen partial pressure $p_{\mathrm{O}_{2}}$, was found to be the most critical parameter. It affects both the growth temperature and the crystallizing phases, as well as the stability of the growth process. While undoped compounds $\mathrm{Ln}_{2} \mathrm{CuO}_{4+\delta}$ grow stable in a pure $\mathrm{O}_{2}$ atmosphere at a pressure of $4-5$ bar, the 
growth at low $p_{\mathrm{O}_{2}}$ is unstable. The opposite situation was found for doped $\mathrm{Ln}_{2-x} \mathrm{Ce}_{\mathrm{x}} \mathrm{CuO}_{4+\delta}$, even for relatively small Ce content $x$. Our growth experiments clearly showed that with increasing Ce content $x$ a decreasing oxygen partial pressure $p_{\mathrm{O}_{2}}$ is required for the optimal growth conditions.

The set of $\mathrm{Nd}_{2-\mathrm{x}} \mathrm{Ce}_{\mathrm{x}} \mathrm{CuO}_{4+\delta}$ crystals was grown in a mixture of $\mathrm{Ar} / \mathrm{O}_{2}$ with $p_{\mathrm{O}_{2}}=0.20$ bar for $x \leq 0.15$ and $p_{\mathrm{O}_{2}} \leq 0.15$ bar for $x \geq 0.15$. The set of $\operatorname{Pr}_{2-\mathrm{x}} \mathrm{Ce}_{\mathrm{x}} \mathrm{CuO}_{4+\delta}$ crystals was also grown in an $\mathrm{Ar} / \mathrm{O}_{2}$ atmosphere with $p_{\mathrm{O}_{2}}=0.20$ bar for $x \leq 0.08$ and $p_{\mathrm{O}_{2}}=0.03$ bar for $x \geq 0.08$. In order to suppress $\mathrm{Cu}$-evaporation from the melt, a pressure of $3-4$ bar was applied. As the evaporation of $\mathrm{Cu}$ is very small compared to the used amount of flux, this loss can be balanced by the flux itself without changing the growth conditions. We note that the change of the flux composition due to the $\mathrm{CuO}$ evaporation can be estimated from the weight loss of the whole system before and after the growth process and does not exceed $2 \%$. The evaporation in $\mathrm{Nd}_{2-\mathrm{x}} \mathrm{Ce}_{\mathrm{x}} \mathrm{CuO}_{4+\delta}$ is slightly higher than in the $\operatorname{Pr}_{2-\mathrm{x}} \mathrm{Ce}_{\mathrm{x}} \mathrm{CuO}_{4+\delta}$ system. A surplus of $1-2 \% \mathrm{CuO}$ in the feed rod composition for compensating this evaporation makes it difficult to control the growth process, which often results in $\mathrm{CuO}$ inclusions and (during the long lasting growth experiments) unstable solvent zones. Apart from the $\mathrm{CuO}$ inclusions, precipitations of $\mathrm{CeO}_{2}$ on microscopic scale and macroscopic clustering of the dopant can only be avoided by means of an appropriate atmosphere with low $p_{\mathrm{O}_{2}}$. Doped crystals grown in pure $\mathrm{O}_{2}$, as it is often suggested in literature $[5,7,8]$, or at too high oxygen partial pressure for the respective Ce content $x$ are inhomogeneous. This results in broad transition curves as well as in ambiguous and varying relations between $T_{\mathrm{c}}$ values and the nominal Ce content $x$. Moreover, a slow decomposition of the compounds over longer time periods (usually several months to several years) is obtained. In order to support the homogeneity of the molten zone, the seed and feed rods are rotated in opposite directions at about $20 \mathrm{rpm}$. The growth rate for all crystals was $0.5 \mathrm{~mm} / \mathrm{h}$ as determined by the external movement of the mirror system. These small growth rates and also small diameters of the grown rods proved to be important for obtaining rods consisting of only a single grain. We note that thinner diameters of the crystallized rod can be obtained by pulling away the feed rod simultaneously during the growth process.

For all crystals the preferred growth direction was along the crystallographic [110] or [100] axis. This is caused by the fact that the grains grow much faster along the CuO-layers than perpendicular to them. In this way only grains with the favorable orientation survive 

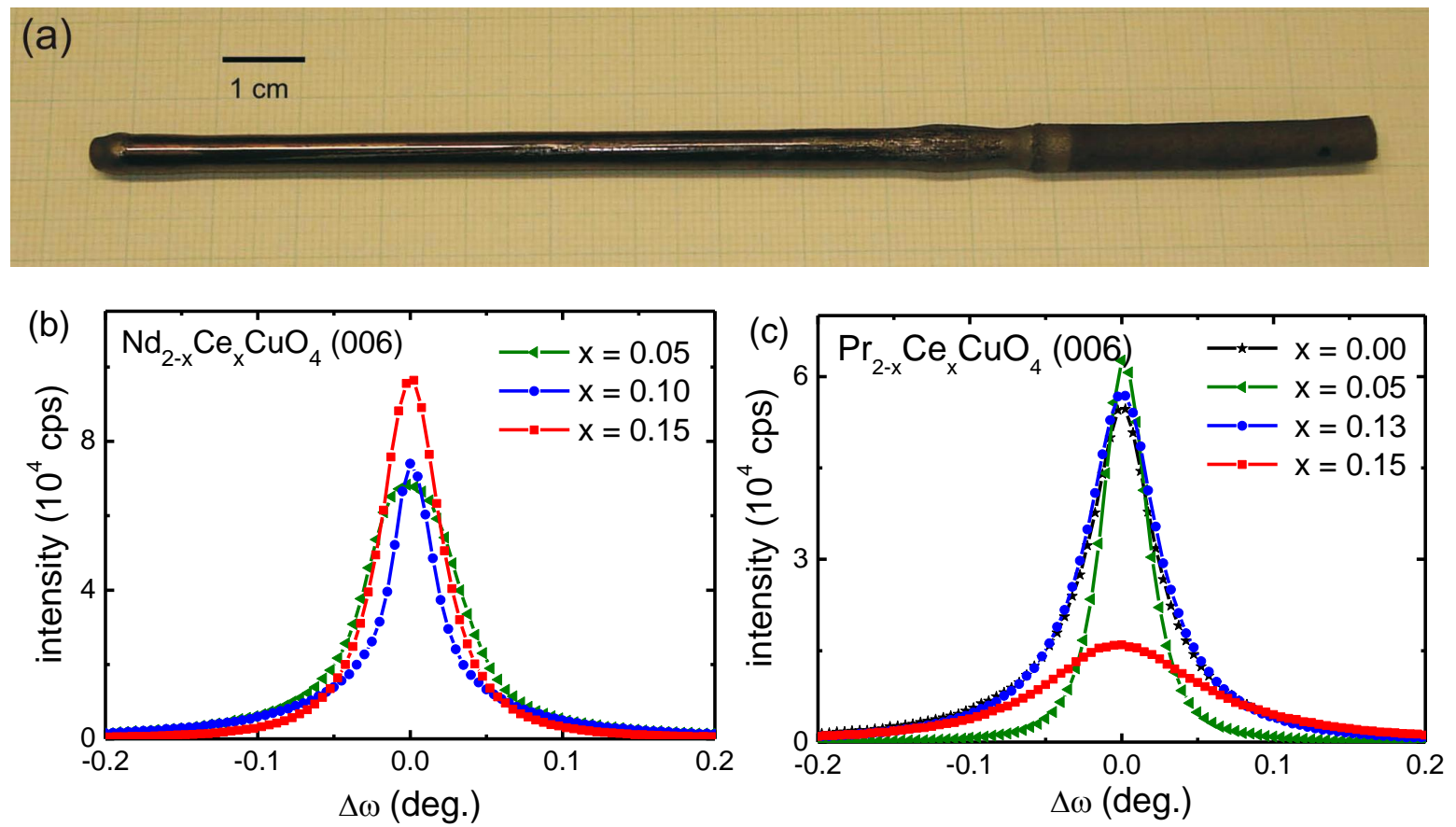

FIG. 1. (a) Picture of a TSFZ-grown $\mathrm{Nd}_{1.85} \mathrm{Ce}_{0.15} \mathrm{CuO}_{4+\delta}$ crystal. (b) and (c) show rocking curves of the (006) peak of $\mathrm{Nd}_{2-\mathrm{x}} \mathrm{Ce}_{\mathrm{x}} \mathrm{CuO}_{4+\delta}$ and $\mathrm{Pr}_{2-\mathrm{x}} \mathrm{Ce}_{\mathrm{x}} \mathrm{CuO}_{4+\delta}$ crystals, respectively. The full width at half maximum (FWHM) for all crystals with different Ce contents is less than $0.08^{\circ}$.

during the growth process. The possibility of the simultaneous growth of several grains with slightly different orientations restricts the use of the samples in experiments, where all these grains have to be extracted and individually oriented. This is for example the case in neutron scattering experiments, where big crystals of several grams are required. A way out would be to influence the growth direction by using a single crystal as seed. However, this has been found difficult, as marginal deviations from the optimal growth conditions at the beginning of the growth process lead to the nucleation of new grains or the crystallization of small amounts of flux material. Fig. 1(a) shows a grown crystal rod of $\mathrm{Nd}_{1.85} \mathrm{Ce}_{0.15} \mathrm{CuO}_{4+\delta}$ with a $c$-oriented facet, which is present on the last $\sim 2 \mathrm{~cm}$ of the rod [left-hand side in Fig. 1(a)] .

\section{B. Crystal Characterization}

The metal composition along and perpendicular to the growth direction of the crystals was controlled by energy dispersive X-ray analysis (EDX). Within the accuracy of the mea- 
surement the grown single crystals exhibit the same chemical composition as the feed rods: no inhomogeneities or gradients of the rare earth concentrations in growth as well as in the radial direction were found. Small deviations in the doping concentration $(x=0.01)$, microscopic precipitations of $\mathrm{Ln}_{2} \mathrm{O}_{3}$ as well as variations in the oxygen distribution within the specimens could not be detected by EDX. More accurate information about the microscopic homogeneity can be obtained from magnetization measurements and from the onset and the shape of superconducting transition curves of different crystals, which are annealed at well defined conditions. The solubility limit of $\mathrm{Ce}$ in $\mathrm{Nd}_{2-\mathrm{x}} \mathrm{Ce}_{\mathrm{x}} \mathrm{CuO}_{4+\delta}$ and $\mathrm{Pr}_{2-\mathrm{x}} \mathrm{Ce}_{\mathrm{x}} \mathrm{CuO}_{4+\delta}$ is found at $x=0.18$ and $x=0.15$, respectively. X-ray analysis confirms the phase purity and the T'-structure of the as-grown crystals. Moreover, the rocking curves of the (006)-peak show a full width at half maximum FWHM $\leq 0.08$ for all doping levels [see Fig. 1(b)]. These values are -to our knowledge- the smallest values reported so far for electron-doped 214compounds. The mosaicity of the crystals is hardly improved by the annealing treatment. However, we can confirm the formation of small amounts of epitaxially grown $(\mathrm{Ln}, \mathrm{Ce})_{2} \mathrm{O}_{3}$ impurity phases $[6,7,9]$ due to the annealing treatment, which is independent from the doping concentration. Rods consisting of two or several grains are separated and oriented before annealing. Bulk superconductivity was verified by specific heat measurements. We emphasize that pieces cut from the inner part of large crystals have the same transition curves $\left(T_{\mathrm{c}}\right.$, $\left.\Delta T_{\mathrm{c}}\right)$ as the whole crystal itself. Thus, core-shell effects can be ruled out. The as-grown and annealed crystals were stable over several years, without changing their physical properties. Crack- and inclusion-free high quality crystals are difficult to cleave. Therefore, for measurements (spectroscopic measurements, transport, etc.) the crystals had to be oriented, cut, and polished in an appropriate way. This time consuming work is a drawback compared to the easily cleaving compounds of the Bi-family and $\mathrm{YBa}_{2} \mathrm{Cu}_{3} \mathrm{O}_{7-\delta}$ which grow in a platelet shape.

\section{ANNEALING TREATMENT}

\section{A. Annealing Procedure}

An annealing treatment of the crystals after the growth process eliminates tension in the crystal and disorder in the metal sublattice. At the same time it removes interstitial oxygen. 
The as-grown $\mathrm{Pr}_{2-\mathrm{x}} \mathrm{Ce}_{\mathrm{x}} \mathrm{CuO}_{4+\delta}$ crystals, grown in an atmosphere of $p_{\mathrm{O}_{2}}=0.03$ bar, are not superconducting, whereas $\mathrm{Nd}_{2-\mathrm{x}} \mathrm{Ce}_{\mathrm{x}} \mathrm{CuO}_{4+\delta}$, grown at the same $p_{\mathrm{O}_{2}}$, shows a broad transition with $T_{\mathrm{c}}=10 \mathrm{~K}$. Unfortunately, the growth process at such low $p_{\mathrm{O}_{2}}$ for $\mathrm{Nd}_{2-\mathrm{x}} \mathrm{Ce}_{\mathrm{x}} \mathrm{CuO}_{4+\delta}$ is not stable enough. Thus, growth atmospheres with higher oxygen partial pressure as described above were used. This results in excess oxygen in the as-grown samples requiring a post-growth annealing treatment.

In the past, many studies concerning the optimal annealing parameters, phase decomposition and oxygen loss have been carried out $[5,7,10,11]$. The general consensus of all these studies is, that bulk superconductivity and sharp transition curves are only realized by a severe reduction treatment of the as-grown samples. In this process a small doping dependent amount of the excess oxygen $\delta=0.02-0.06$ is removed. Nevertheless, fundamental questions regarding the onset, shape and doping interval of the superconducting dome, the absolute value of the oxygen content of the as-grown and reduced samples, the role of additional oxygen in the compound, and the reversibility of the reduction step have not yet been settled and currently are controversially discussed. In order to verify the different results, we annealed the samples in the following way: As-grown single crystals were annealed for $t_{\mathrm{ann}}=20 \mathrm{~h}$ in a flow of pure $\operatorname{Ar} 4.8\left(\mathrm{O}_{2} \leq 3 \mathrm{ppm}\right)$ at constant temperatures close to the stability limit. The stability limit for this annealing treatment depends on the Ce content as well as on the rare earth element $L n$ and is shown in Fig. 2.

For the annealing step, the crystals were enclosed in polycrystalline crucibles of the same material in order to provide a homogeneous environment and to protect the crystal surface. After the treatment at high temperatures the crystals were cooled down to room temperature at moderate cooling rates. The crystals were not quenched, since the intention was to remove the excess oxygen but not to freeze-in a specific $\mathrm{O}_{2}$ ordering state. In this way we avoided additional tension in the metallic sublattice which could occur upon quenching. This procedure was applied to all doped crystals of both compounds. Depending on the Ce content $x$, the crystals exhibit sharp transition curves as shown in Fig. 3. It has been often reported in literature $[5,7]$ that the annealing treatment depends on the sample size. Moreover, it was suggested that a subsequent additional annealing step after the reduction step, which is then performed in pure $\mathrm{O}_{2}$ at moderate temperatures of $500-600^{\circ} \mathrm{C}$ for $10 \mathrm{~h}$ increases $T_{\mathrm{c}}$. However, for our crystals with masses ranging from 50 to $250 \mathrm{mg}$ we do not see any size effect of the transition curves. This observation confirms that the 


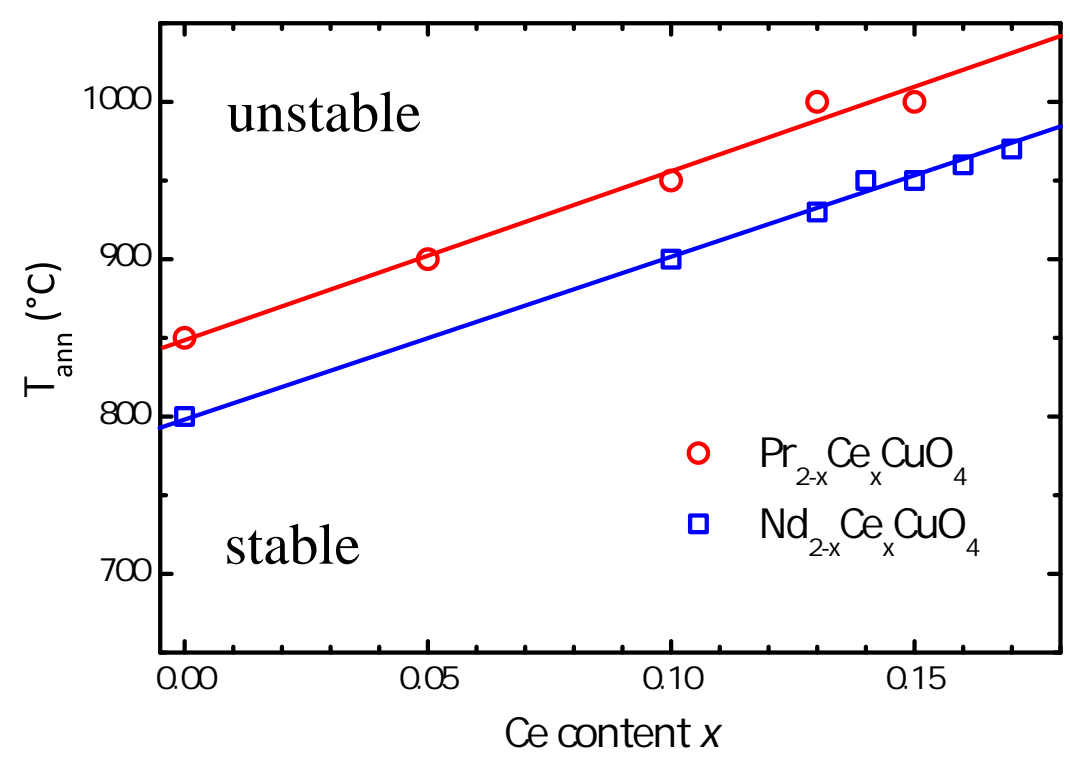

FIG. 2. Doping dependence of the maximum annealing temperature $T_{\text {ann }}$ for $\mathrm{Nd}_{2-\mathrm{x}} \mathrm{Ce}_{\mathrm{x}} \mathrm{CuO}_{4+\delta}$ and $\operatorname{Pr}_{2-x} \mathrm{Ce}_{\mathrm{x}} \mathrm{CuO}_{4+\delta}$. The lines are guides to the eye. All samples are annealed in a flow of pure $\operatorname{Ar} 4.8\left(\mathrm{O}_{2} \leq 3 \mathrm{ppm}\right)$ for $t_{\mathrm{ann}}=20 \mathrm{~h}$.

oxygen diffusion coefficient $D(T)$ and diffusion lengths $l \sim \sqrt{D(T) \cdot t_{\text {ann }}}$ are large enough at the annealing temperatures used in our experiments. Moreover, a second, short annealing treatment at moderate temperatures in pure $\mathrm{O}_{2}$ complicates the control of the oxygen content and the microscopic homogeneity of the crystal, as $D(T)$ might be completely different at the different annealing steps at high and moderate temperatures. For example, it is well known from diffusion studies on $\mathrm{YBa}_{2} \mathrm{Cu}_{3} \mathrm{O}_{7-\delta}$ and $\mathrm{RE}-123$ single crystals, that $D(T)$ varies over several orders of magnitude. Since there are no reliable studies of the kinematics of the oxygen diffusion process for the 214-crystals, results of annealing experiments under different atmospheres, temperatures and time scales are speculative. We also note that studies on large ceramic samples give misleading results, as the in- and out-diffusion of oxygen is mainly governed by grain boundaries, micro-cracks and other imperfections of the specimen.

\section{B. Absolute Value of the Oxygen Concentration}

Thermogravimetric experiments and subsequent x-ray powder diffraction on high quality as-grown single crystals are performed in order to estimate the absolute oxygen content $4+\delta$ per formula unit. The absolute oxygen concentration is required for an accurate 

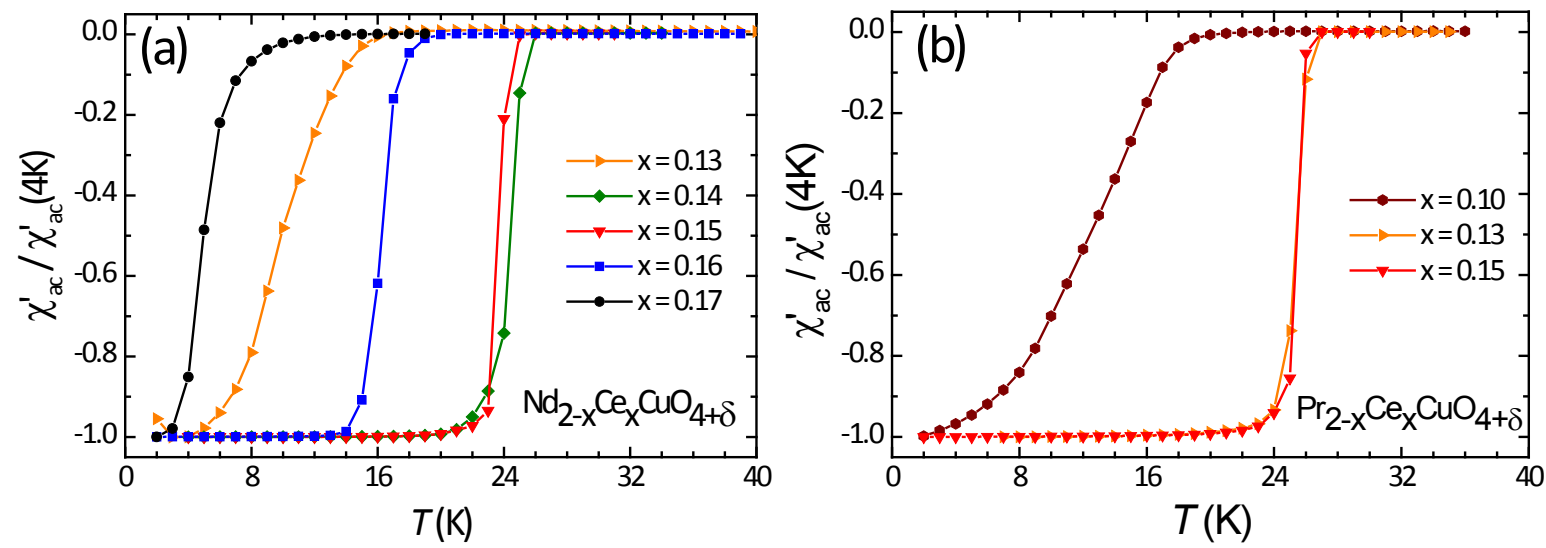

FIG. 3. Ac-susceptibility of $\mathrm{Nd}_{2-\mathrm{x}} \mathrm{Ce}_{\mathrm{x}} \mathrm{CuO}_{4+\delta}$ (a) and $\mathrm{Pr}_{2-\mathrm{x}} \mathrm{Ce}_{\mathrm{x}} \mathrm{CuO}_{4+\delta}$ (b) single crystals plotted versus temperature for samples with different Ce contents. For a better comparison the measured diamagnetic susceptibilities are normalized to their low temperature values. Note, that the sample mass ranges from 100 to $250 \mathrm{mg}$. For $\mathrm{Nd}_{1.85} \mathrm{Ce}_{0.15} \mathrm{CuO}_{4+\delta}$ we obtain $T_{\mathrm{c}}=23.6 \mathrm{~K}$ and $\Delta T_{\mathrm{c}}=1.3 \mathrm{~K}$, whereas for $\operatorname{Pr}_{1.85} \mathrm{Ce}_{0.15} \mathrm{CuO}_{4+\delta}$ we find $T_{\mathrm{c}}=25.5 \mathrm{~K}$ and $\Delta T_{\mathrm{c}}=1.4 \mathrm{~K}$.

determination of the oxygen loss during the annealing treatment from the measured mass difference between as-grown and annealed samples. As the oxygen non-stoichiometry in the 214-compounds is very small, only crack- and inclusion-free single crystals of $100-150 \mathrm{mg}$ mass were used for these experiments in order to avoid misleading results. Systematic errors coming from the experimental setup are minimized by recording a base line ahead of each measurement. The oxygen concentration is calculated from the measured weight loss during the reduction in a flow of forming gas with composition $\mathrm{H}_{2} / \mathrm{N}_{2}=15 / 85$ for $1 \mathrm{~h}$ at $1000^{\circ} \mathrm{C}$. The crystals decompose according to the following equation, where $L n=\mathrm{Nd}$, $\operatorname{Pr}$ :

$$
2 \mathrm{Ln}_{2-x} \mathrm{Ce}_{x} \mathrm{CuO}_{4+\delta} \rightarrow(2-x) \mathrm{Ln}_{2} \mathrm{O}_{3}+2 x \mathrm{CeO}_{2}+2 \mathrm{Cu}^{\text {ele }}+\left(1+\delta-\frac{x}{2}\right) \mathrm{O}_{2} .
$$

Undoped as-grown crystals show a nearly stoichiometric oxygen content. Upon increasing the Ce content $x$ the oxygen surplus $\delta$ increases slightly and has values of about 0.03 for optimally doped $\mathrm{Nd}_{1.85} \mathrm{Ce}_{0.15} \mathrm{CuO}_{4+\delta}$ and $\operatorname{Pr}_{1.85} \mathrm{Ce}_{0.15} \mathrm{CuO}_{4+\delta}$. Note, that the absolute value depends on the oxygen partial pressure $p_{\mathrm{O}_{2}}$ used during the crystal growth. This might also be an explanation of different values in literature reported for the oxygen non-stoichiometry and the oxygen loss during annealing, as the crystals are grown in different atmospheres. For crystals with Ce concentrations $x$ within the superconducting dome the oxygen loss amounts to 0.02 for $\mathrm{Nd}_{2-\mathrm{x}} \mathrm{Ce}_{\mathrm{x}} \mathrm{CuO}_{4+\delta}$ and $\mathrm{Pr}_{2-\mathrm{x}} \mathrm{Ce}_{\mathrm{x}} \mathrm{CuO}_{4+\delta}$. Thus, the annealed samples 
have a stoichiometric number of $4+\delta=4.01$ for oxygen, which hardly differs from the nominal value of 4.0 within the uncertainty of the experiment. Therefore, as discussed in more detail below, oxygen does not play an important role regarding the doping level and we can set $n=x$. Here, $n$ is the carrier concentration per $\mathrm{Cu}$-ion and $x$ is the Ce concentration.

\section{DOPING DEPENDENT PHASE DIAGRAM}

\section{A. The Superconducting Dome}

Fig. 4 shows the transition temperatures as a function of the Ce content $x$ for both electron-doped 214-compounds discussed in this paper. We recall that in the hole-doped cuprates superconductivity appears within a broad doping interval of $p=[0.05,0.27]$ and $T_{\mathrm{c}} \propto\left(p-p_{\text {opt }}\right)^{2}$, where $p_{\text {opt }}$ is the optimum doping level corresponding to the maximum $T_{\mathrm{c}}$ value [12]. In contrast, for the electron-doped compounds $\mathrm{Nd}_{2-\mathrm{x}} \mathrm{Ce}_{\mathrm{x}} \mathrm{CuO}_{4+\delta}$ and $\mathrm{Pr}_{2-\mathrm{x}} \mathrm{Ce}_{\mathrm{x}} \mathrm{CuO}_{4+\delta}$ the superconducting dome is restricted to a much smaller doping range and does in general not show a quadratic dependence $T_{\mathrm{c}} \propto\left(n-n_{\mathrm{opt}}\right)^{2}$. As shown in Fig. 4, for the $\mathrm{Nd}_{2-\mathrm{x}} \mathrm{Ce}_{\mathrm{x}} \mathrm{CuO}_{4+\delta}$ system superconductivity abruptly sets in at $n \simeq x=0.13$ and $T_{\mathrm{c}}$ jumps up to $T_{\mathrm{c}, \max } n \simeq x=0.146$. In the overdoped region the $T_{\mathrm{c}}(x)$ dependence can be well described by the empirical quadratic function (solid line in Fig. 4)

$$
\frac{T_{\mathrm{c}}}{T_{\mathrm{c}, \max }}=1-1320(x-0.146)^{2}
$$

with $T_{\mathrm{c}, \max }=25.1 \mathrm{~K}$. According to this empirical curve, $T_{\mathrm{c}}$ becomes zero at $x=0.118$ and $x=0.173$. A comparison of the empirical curve with the experimental results in the underdoped region reveals a slight asymmetry of the superconducting dome in the $\mathrm{Nd}_{2-\mathrm{x}} \mathrm{Ce}_{\mathrm{x}} \mathrm{CuO}_{4+\delta}$ system. However, more crystals with even finer variations in the Ce concentration in the range $[0.12,0.13]$ are required to clarify the onset point and the sudden occurrence of superconductivity with $T_{\mathrm{c}}$ values of about $0.4 T_{\mathrm{c}, \max }$ at $x=0.13$. From the doping point of view the overdoped region can be controlled in a better way. Notably, the whole superconducting region down to $T_{\mathrm{c}}=0$ can be probed, as a solubility limit of $x=0.18$ is found for $\mathrm{Ce}$ in the $\mathrm{Nd}_{2-\mathrm{x}} \mathrm{Ce}_{\mathrm{x}} \mathrm{CuO}_{4+\delta}$ system. In this context the width of the transition curves as a measure of the quality of the crystals has to be discussed. It is clear, that crys-

tals near optimal doping show the sharpest transition curves, since slight variations in the doping of the specimen do not strongly affect $T_{\mathrm{c}}$. In the overdoped region, the crystals are 
very sensitive to small doping variations due to the steep slope of the $T_{\mathrm{c}}(x)$ curve defining the superconducting dome. For example, for a sample with nominal doping $x=0.17$ and a spatial variation in the doping level as small as $\Delta x=0.002$, a transition width of $\Delta \geq 3 \mathrm{~K}$ is expected. In the underdoped region the situation is even more complicated and it is not clear whether inhomogeneities of the crystals or other effects are responsible for the observed broad transition curves.

In the $\operatorname{Pr}_{2-\mathrm{x}} \mathrm{Ce}_{\mathrm{x}} \mathrm{CuO}_{4+\delta}$ system, the overdoped region cannot be reliably probed due to precipitations of the dopant. Therefore, the comparison of the superconducting phase diagram is restricted to the optimal and underdoped regions. Comparing the $\operatorname{Pr}_{2-\mathrm{x}} \mathrm{Ce}_{\mathrm{x}} \mathrm{CuO}_{4+\delta}$ and $\mathrm{Nd}_{2-\mathrm{x}} \mathrm{Ce}_{\mathrm{x}} \mathrm{CuO}_{4+\delta}$ system, we have to point to two common properties: first, the optimum doping level $x=0.15$ is about the same and, second, both systems show an abrupt onset of superconductivity in the underdoped region. For $\operatorname{Pr}_{2-\mathrm{x}} \mathrm{Ce}_{\mathrm{x}} \mathrm{CuO}_{4+\delta}$, this onset is found at $x=0.10$. However, there are also pronounced differences in the $T_{\mathrm{c}}(x)$ dependence of both systems. It is evident from Fig. 4 that the $\mathrm{Pr}_{2-\mathrm{x}} \mathrm{Ce}_{\mathrm{x}} \mathrm{CuO}_{4+\delta}$ system shows a much broader $T_{\mathrm{c}}(x)$ dependence in the underdoped regime. This difference in the shape of the superconducting dome is most likely the result of material specific issues and is a general problem when comparing electron-doped compounds. Therefore, in the future one main task should be the elimination of material specific factors in order to merge the different phase diagrams of the electron-doped compound into a general one.

\section{B. Role of Oxygen}

The precise experimental control of the oxygen concentration is one of the main difficulties when preparing 214-compounds. It is challenging to tune and determine the small oxygen variations in a crystal in a non-destructive manner. Therefore, many and partly contradicting statements concerning the oxygen occupation of the different sites in the crystal structure of as-grown and annealed crystals exist [13-15]. Oxygen is often believed to act as co-dopant in such reports. In order to clarify whether or not oxygen non-stoichiometry may act as a second doping channel, an overdoped $\mathrm{Nd}_{1.84} \mathrm{Ce}_{0.16} \mathrm{CuO}_{4+\delta}$ high quality single crystal was used for oxygenation experiments. Strongly overdoped crystals are expected

to be extremely sensitive to oxygenation experiments, because of the steep slope of the superconducting dome (cf. Fig. 4) in this regime. 

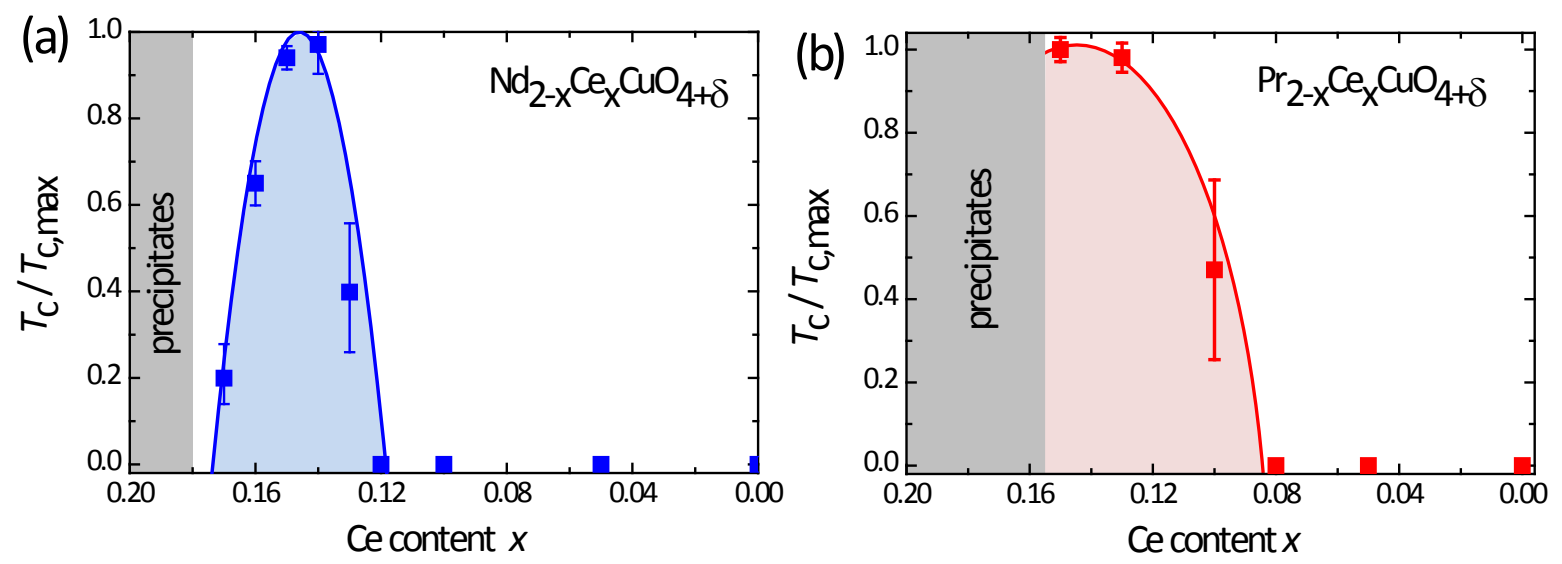

FIG. 4. Normalized transition temperature of $\mathrm{Nd}_{2-\mathrm{x}} \mathrm{Ce}_{\mathrm{x}} \mathrm{CuO}_{4+\delta}$ (a) and $\mathrm{Pr}_{2-\mathrm{x}} \mathrm{Ce}_{\mathrm{x}} \mathrm{CuO}_{4+\delta}$ (b) single crystals plotted versus the Ce concentration $x$. The $T_{\mathrm{c}}$ values have been derived from the transition curves in Fig. 3. The data points mark the $T_{\mathrm{c}}$ values where the resistivity dropped to $50 \%$ of its normal state value. The vertical bars indicated the transition widths $\Delta T_{\mathrm{c}}$ in which the resistivity dropped from $90 \%$ to $10 \%$. In (a) the experimental data of the overdoped regime are fitted by the quadratic function resulting in the empirical relation (1). The superconducting dome is asymmetric with an abrupt onset at $x=0.13$, an expected maximum at $x=0.146$ and a steady decrease towards zero before the solubility limit at $x=0.18$ is reached. For the $\operatorname{Pr}_{2-\mathrm{x}} \mathrm{Ce}_{\mathrm{x}} \mathrm{CuO}_{4+\delta}$ system shown in (b) the overdoped regime cannot be probed due to precipitations. The optimal doping is found at $x=0.15$ and the underdoped region extends to $x=0.10$, where superconductivity abruptly sets in.

In a simple ionic model of the electron-doped 214-compounds the oxygen contribution to the electron concentration $n$ per $\mathrm{Cu}$-ion is given by the relation

$$
n=x-2 \delta .
$$

Here, the valences for the constituents are assumed to be $\mathrm{Ce}=4+, \mathrm{Ln}=3+, \mathrm{O}=2-, \mathrm{Cu}=$ $\nu$. The formal valency of $\mathrm{Cu}$ is $\nu=2-n=2-x+2 \delta$. In this picture the presence of any excess oxygen with amount $\delta$ lowers the charge carrier concentration $n$ and, hence, compensates the carrier concentration induced by the Ce content $x$. By starting with a reduced overdoped $\mathrm{Nd}_{1.84} \mathrm{Ce}_{0.16} \mathrm{CuO}_{4.01}$ crystal, the optimally doped and underdoped regions of the superconducting dome should be reached easily by appropriate oxygenation. In Fig. 5(a) the change of the transition curve by an oxygen variation of $\Delta \delta=+0.005$ is presented. Clearly, $T_{\mathrm{c}}$ is lowered by $1 \mathrm{~K}$ and the absolute value of the ac-response at $2 \mathrm{~K}$ is reduced by a factor of 

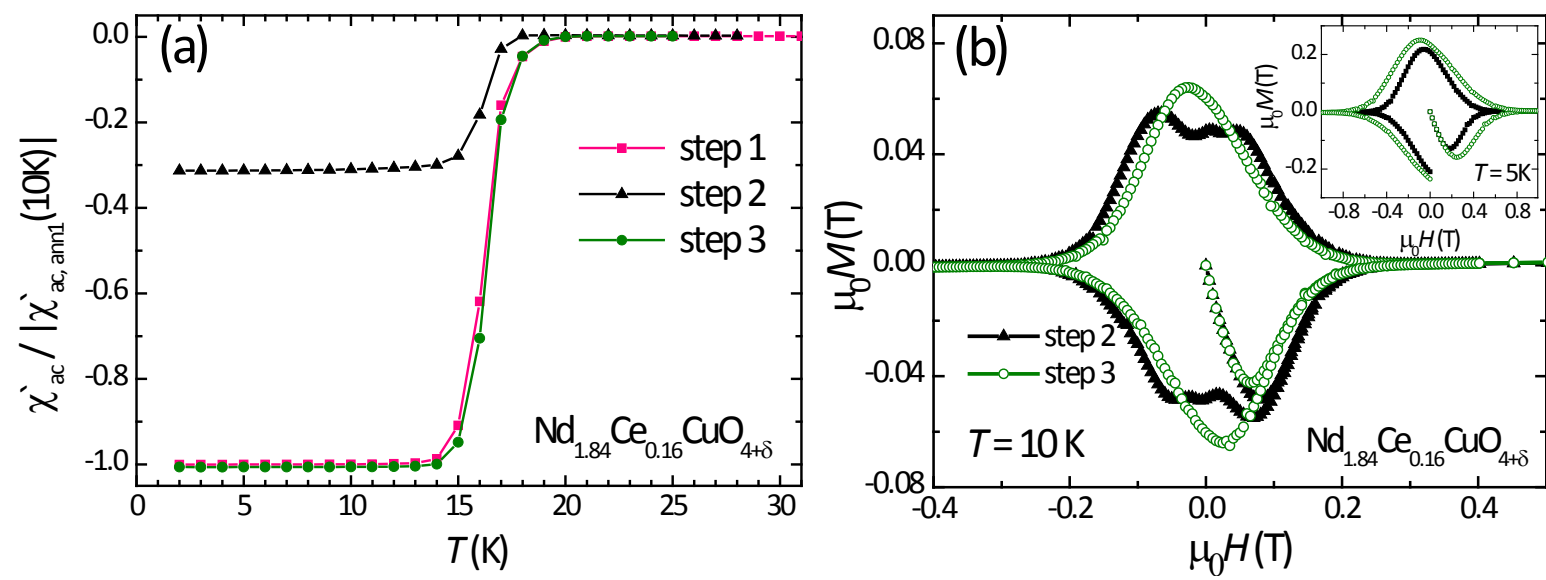

FIG. 5. (a) Temperature dependence of the normalized ac susceptibility recorded for a $c$-oriented $\mathrm{Nd}_{1.84} \mathrm{Ce}_{0.16} \mathrm{CuO}_{4+\delta}$ crystal of $51.5 \mathrm{mg}$ after different steps of an annealing experiments. Step 1: Reduction of the as-grown sample in flowing of $\operatorname{Ar}(4.8)$ at $960^{\circ} \mathrm{C}$ for $20 \mathrm{~h}(\Delta \delta=-0.018)$. Step 2 : Oxygenation of the reduced sample in a flowing $\mathrm{O}_{2} / \mathrm{Ar}=0.1 \%$ at $750^{\circ} \mathrm{C}$ for $70 \mathrm{~h}(\Delta \delta=+0.005)$. Step 3: Repetition of step 1 with the same parameters $(\Delta \delta=-0.006)$. The ac-susceptibility curves confirm the reversibility of the annealing steps. The transition curve after oxygenation shows $T_{\mathrm{c}}$ reduced by $1 \mathrm{~K}$ and a smaller (by a factor of 3 ) diamagnetic susceptibility. (b) Magnetization curves recorded at $5 \mathrm{~K}$ (inset) and $10 \mathrm{~K}$ after the annealing steps 2 and 3 . After partial oxygenation the crystal shows a broader magnetization curve with irregularities. This fluctuating fishtail effect, which appears/disappears with oxygenation/reduction is a clear signature for oxygen induced disorder, which gradually destroys superconductivity.

about 3. This is in complete contradiction to our simple model and the measured phase diagram. According to $n=x-2 \delta$, for the $\mathrm{Nd}_{1.84} \mathrm{Ce}_{0.16} \mathrm{CuO}_{4.01}$ sample the carrier concentration $n$ is expected to change from $n=0.14$ to $n=0.13$ on going from $\mathrm{O}_{4.010}$ to $\mathrm{O}_{4.015}$ and, thus, an increase of $T_{\mathrm{c}}$ towards $T_{\mathrm{c}, \max }$ is expected. A subsequent standard reduction step removes the inserted oxygen again $(\Delta \delta=-0.006)$ and the crystal shows the same sharp transition curve as before oxygenation. From this annealing experiment we arrive at two conclusions: First, the metal sublattice remains unaffected by carefully performed oxygenation experiments. In particular, no irreversible microscopic $\mathrm{CeO}_{2}$-precipitations or rearrangements occur, which could be responsible for the changes in the transition curves. Second, the oxygen surplus produces no significant doping effect as expected from the simple ionic model presented above. Therefore, the use of the identity $n=x$ is well justified. We note, however, 

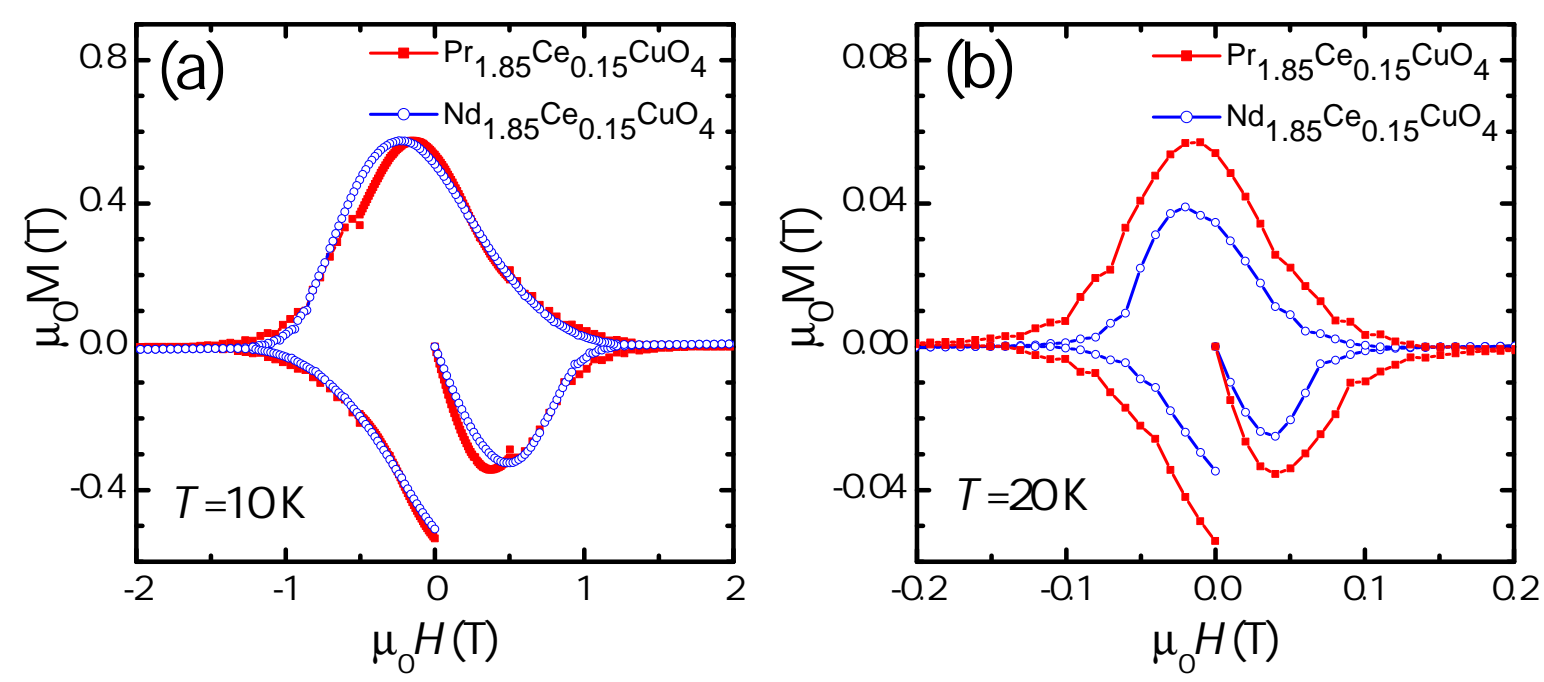

FIG. 6. Magnetization curves of a $c$-oriented (a) $\mathrm{Nd}_{1.85} \mathrm{Ce}_{0.15} \mathrm{CuO}_{4+\delta}$ (182 mg) and (b) $\operatorname{Pr}_{1.85} \mathrm{Ce}_{0.15} \mathrm{CuO}_{4+\delta}(100 \mathrm{mg})$ single crystal. The corresponding transition curves are shown in Fig. 3. No anomalies are detected, thereby confirming the microscopic homogeneity of the crystals.

that there is a strong oxygen induced microscopic disorder, which suppresses very effectively superconductivity and can be detected by magnetization measurements (fishtail effect, see Fig. 5(b)). In our well reduced single crystals such irregularities in the magnetization data have not been observed (see also Fig. 6).

Since there is only a single impurity site available for oxygen occupation or reduction in the 214-compounds, we believe, that the population/depopulation of this apical $\mathrm{O}(3)$ site is closely related to the appearance of superconductivity. The presence of apical oxygen may cause additional distortions and strain within the $\mathrm{CuO}_{2}$ plane, preventing the evolution of the superconducting state independent of the doping $x$. This picture is consistent with the experimental observations of non-superconducting or weakly superconducting as-grown crystals depending on the used $p_{\mathrm{O}_{2}}$ during crystal growth, the evolution/disappearence of superconductivity after removal/incorporation of additional oxygen as well as with recent transport measurements on $\operatorname{Pr}_{2-x} \mathrm{Ce}_{x} \mathrm{CuO}_{4+\delta}$ thin films [13]. We do not believe, that the oxygen reduction occurs mainly in the $\mathrm{CuO}_{2}$ planes while the apical site occupation remains unchanged [15], as in this case the oxygen variation should trigger a doping effect. 


\section{SHUBNIKOV-DE HAAS OSCILLATIONS}

The very high quality of $\mathrm{Nd}_{2-\mathrm{x}} \mathrm{Ce}_{\mathrm{x}} \mathrm{CuO}_{4+\delta}$ single crystals obtained following the procedure described above is clearly demonstrated by the observation of magnetic quantum oscillations in their interlayer resistivity for doping levels $x=0.15,0.16$, and 0.17 [16]. A particular prerequisite for the observation of magnetic quantum oscillations is that the mean free path $\ell$ of the charge carriers be at least comparable to the radius $r_{B}=p_{F} / e B$ of the cyclotron orbit, where $p_{F}$ is the Fermi momentum perpendicular to magnetic field $\mathbf{B}$ and $e$ the elementary charge. For superconducting cuprates, $r_{B}$ is a few hundred ångströms in experimentally available magnetic fields, thus imposing stringent requirements on the crystal quality. Although $\mathrm{Nd}_{2-\mathrm{x}} \mathrm{Ce}_{\mathrm{x}} \mathrm{CuO}_{4+\delta}$ is a complex solid solution system, this requirement is fulfilled for our high-quality single crystals.

The crystals used in our high magnetic field experiments showed consistent temperatures and widths of the superconducting transition for each doping with those presented in Fig. 3(a). The resistivity ratios, $\rho(273 K) / \rho\left(T_{0}\right)=5.6,5.2$, and 9.7 , for $x=0.15,0.16$, and 0.17 , respectively ( $T_{0}$ is the superconducting onset temperature), also indicate high crystal quality.

Fig. 7(a) shows the oscillatory component of the interlayer resistivity of the $x=0.17$ sample at $T=2.7 \mathrm{~K}$. The data is obtained from a raw $R(B)$ curve by subtracting a monotonic background. The oscillations are periodic in the $1 / B$ scale, their positions being independent of temperature [16]. Such behavior is characteristic of the Shubnikov-de Haas $(\mathrm{SdH})$ effect originating from the Landau quantization of the electron spectrum of a metal in a high magnetic field [17]. The oscillation frequency, $F_{0.17}=(10950 \pm 100) \mathrm{T}$ is directly related to the area of the cyclotron orbit on the cylindrical Fermi surface: $A_{0.17}=2 \pi e F_{0.17} / \hbar=(1.04 \pm 0.01) \times 10^{20} \mathrm{~m}^{-2}$. This corresponds to $41.4 \%$ of the first Brillouin zone area, in excellent agreement with the predictions of band structure calculations [18] and results of angle-resolved photoemission spectroscopy (ARPES) [19, 20].

The samples with lower doping, $x=0.15$ and 0.16, also show $\mathrm{SdH}$ oscillations (see Fig. 7(b)), however, at much lower frequencies, $F_{0.15}=(290 \pm 10) \mathrm{T}$ and $F_{0.16}=(280 \pm 10) \mathrm{T}$, respectively. The corresponding Fermi surface cross-section amounts to only $\approx 1.1 \%$ of the Brillouin zone area. Such a drastic change of the SdH frequency indicates a reconstruction of the Fermi surface due to a broken translational symmetry. It can be explained by introducing 

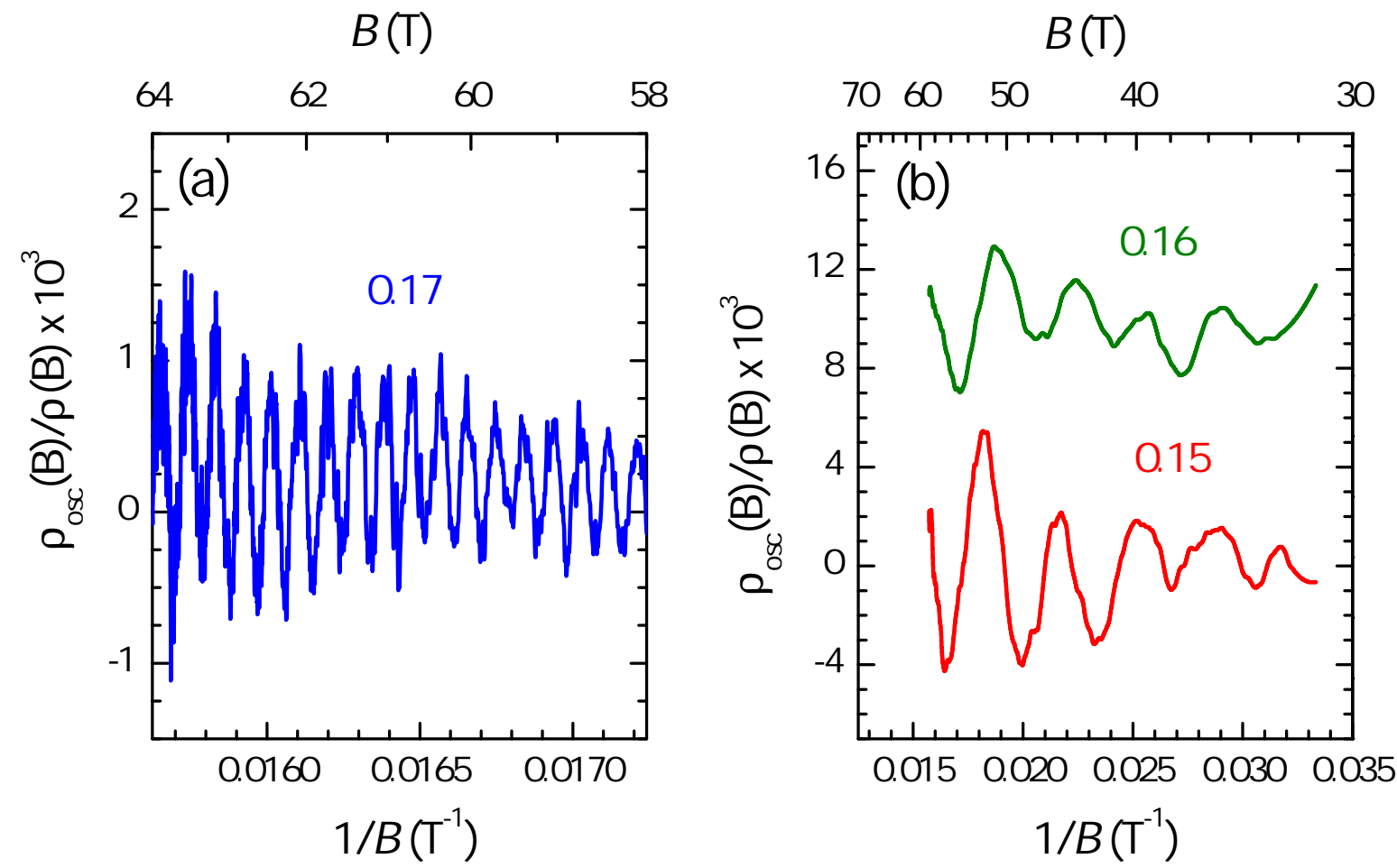

FIG. 7. Shubnikov-de Haas oscillations in $\mathrm{Nd}_{2-\mathrm{x}} \mathrm{Ce}_{\mathrm{x}} \mathrm{CuO}_{4+\delta}$ crystals with (a) $x=0.17$, and (b) $x=0.15$ and 0.16 , at a magnetic field applied perpendicular to $\mathrm{CuO}_{2}$ layers. Note that the oscillation frequency for $x=0.17$ is 30 times higher than for the lower doping levels.

a $(\pi / a, \pi / a)$ density-wave potential $[16,21]$. The slow oscillations are consistent with the size of small hole pockets formed at the new Brillouin zone boundary as a result of folding the original large Fermi surface.

Similar results revealing small closed pockets of a reconstructed Fermi surface have been obtained from quantum oscillation experiments on p-underdoped $\mathrm{YBa}_{2} \mathrm{Cu}_{3} \mathrm{O}_{6.5}[22-$ 24] and $\mathrm{YBa}_{2} \mathrm{Cu}_{4} \mathrm{O}_{8}[25,26]$. However, by contrast to the latter compounds, in the n-doped $\mathrm{Nd}_{2-\mathrm{x}} \mathrm{Ce}_{\mathrm{x}} \mathrm{CuO}_{4+\delta}$ the ordering potential is manifest already on the overdoped side of the phase diagram, at $x=0.16$. Moreover, the observation of fast oscillations at $x=0.17$ does not unambiguously rule out the possibility of the superlattice at this doping. Indeed, our most recent data on samples with $x=0.17$ [27] show evidence of magnetic breakdown, suggesting the Fermi surface to be reconstructed even at this high doping level. Further work on magnetic quantum oscillations is in progress, in order to verify this suggestion and obtain more information about the Fermi surface and its dependence on the carrier concentration in the electron-doped superconductors. 


\section{SUMMARY}

We have grown high quality single crystals of the electron-doped cuprate superconductors $\mathrm{Nd}_{2-\mathrm{x}} \mathrm{Ce}_{\mathrm{x}} \mathrm{CuO}_{4+\delta}$ and $\mathrm{Pr}_{2-\mathrm{x}} \mathrm{Ce}_{\mathrm{x}} \mathrm{CuO}_{4+\delta}$. We discuss the optimal growth parameters and annealing conditions as a function of Ce content $x$ required to achieve such crystals. We show that the oxygen partial pressure of the growth atmosphere has to be reduce with increasing $x$ in order to avoid microscopic $\mathrm{CeO}_{2}$ precipitations. We also present an optimized annealing process resulting in narrow transition curves. In the $\mathrm{Nd}_{2-\mathrm{x}} \mathrm{Ce}_{\mathrm{x}} \mathrm{CuO}_{4+\delta}$ system the whole phase diagram can be probed with high quality single crystals, whereas in $\operatorname{Pr}_{2-\mathrm{x}} \mathrm{Ce}_{\mathrm{x}} \mathrm{CuO}_{4+\delta}$ the overdoped regime is inaccessible due to the lower solubility limit of Ce. Here, the system $\mathrm{La}_{1} \mathrm{Pr}_{1-x} \mathrm{Ce}_{x} \mathrm{CuO}_{4+\delta}$ might be an adequate alternative. The microscopic oxygen distribution can be checked by magnetic measurements. The as-grown doped crystals exhibit an excess oxygen concentration of $\delta \approx 0.03$, which is nearly completely removed after annealing. The oxygen reduction is reversible and the removed oxygen comes primary from the apical site. This apical oxygen governs the evolution of superconductivity by virtue of induced disorder in the $\mathrm{CuO}_{2}$ planes. The additional oxygen has only a minor doping effect. The obtained crystals reach a perfection that quantum oscillations can be observed on such samples.

\section{ACKNOWLEDGEMENTS}

The authors are very grateful to R. Gross for numerous stimulating and fruitful discussions. The work was supported the German Research Foundation via the Research Unit FOR 538. We also acknowledge support by EuroMagNET II under the EC contract No. 228043.

[1] Y. Tokura, H. Takagi, S. Uchida, Nature 337, 345 (1989).

[2] A. Erb, E. Walker, R. Fluekiger, Physica C 258, 9 (1996).

[3] A.N. Maljuk, A.A. Jokhov, I.G. Naumenko, I.K. Bdikin, S.A. Zver'kov, G.A. Emel'chenko, Physica C 329, 51 (2000).

[4] K. Oka, H. Unoki, Jpn. J. Appl. Phys. 28, L937 (1989).

[5] K. Kurahashi, H. Matsushita, M. Fujita, K. Yamada, J. Phys. Soc.Jpn. 71, 910 (2002). 
[6] M. Matsuura, P. Dai, H.J. Kang, J.W. Lynn, D.N. Argyriou, K. Prokes, Y. Onose, Y. Tokura, Phys. Rev. B 68, 144503 (2003).

[7] P.K. Mang, S. Larochelle, A. Mehta, O.P. Vajk, A.S. Erickson, L. Lu, W.J.L. Buyers, A.F. Marshall, Phys. Rev. B 70, 094507 (2004).

[8] T. Uefuji, S. Kuroshima, M. Fujita, and K. Zamada, Physica C 392-396,189 (2003).

[9] H.J. Kang, P. Dai, H.A. Mook, D.N. Argyriou, V. Sikolenko, J.W. Lynn, Y. Kurita, S. Seiki, Y. Ando, Phys. Rev. B 71, 214512 (2005).

[10] H. Shibata, K. Oka, S. Kashiwaya, H. Yamaguchi, Physica C 357, 363 (2001).

[11] J.S. Kim, D.R. Gaskell, Physica C 209, 381 (1993).

[12] J.L. Tallon, C. Bernhard, H. Shaked, R.L. Hitterman, J.D. Jorgensen, Phys. Rev. B 751, 12911 (1995).

[13] J.S. Higgins, Y. Dagan, M.C. Barr, B.D. Weaver, R.L. Greene, Phys. Rev. B 73, 104510 (2006).

[14] P.G. Radaelli, J.D. Jorgensen, A.J. Schultz, J.L. Peng, R.L. Greene, Phys. Rev. B 49, 15322 (1994).

[15] P. Richard, G. Riou, I. Hetel, S. Jandl, M. Poirier, P. Fournier, Phys. Rev. B 70, 0645131 (2004).

[16] T. Helm, M. V. Kartsovnik, M. Bartkowiak, N. Bittner, M.Lambacher, A. Erb, J. Wosnitza, and Gross, Phys. Rev. Lett. 103, 57002 2009).

[17] D. Shoenberg, Magnetic Oscillations in Metals (Cambridge University Press, Cambridge, 1984).

[18] S. Massidda, N. Hamada, J. Yu, and A. J. Freeman, Physica C 157, 571 (1989).

[19] H. Matsui, T. Takahashi, T. Sato, K. Terashima, H. Ding, T. Uefuji, and K. Yamada, Phys. Rev. B 75, 224514 (2007).

[20] P. N. Armitage, F. Ronning, D. H. Lu, C. Kim, A. Damascelli, K. M. Shen, D. L. Feng, H. Eisaki, Z.-H. Shen, P.K. Mang et al., Phys. Rev. Lett. 88, 257001 (2002).

[21] J. Eun, X. Jia, and S. Chakravarty, Arxiv:0912.0728; unpublished.

[22] N. Doiron-Leyraud, C. Proust, D. LeBoeuf, J. Levallois, J.-B. Bonnemaison, R. Liang, D. A. Bonn, W. N. Hardy, and L Taillefer, Nature 447, 565 (2007).

[23] C. Jaudet, D. Vignolles, A. Audouard, J. Levallois, D. LeBoeuf, N. Doiron-Leyraud, B. Vignolle, M. Nardone, A. Zitouni, R. Liang, et al., Phys. Rev. Lett. 100, 187005 (2008). 
[24] S. E. Sebastian, N. Harrison, E. Palm, T. P. Murphy, C. H. Mielke, R. Liang, D. A. Bonn, W. N. Hardy, and G. G. Lonzarich, Nature 454, 200 (2008).

[25] E. A. Yelland, J. Singleton, C. H. Mielke, N. Harrison, F. F. Balakirev, B. Dabrowski, and J. R. Cooper, Phys. Rev. Lett. 100, 047003 (2008).

[26] A. F. Bangura, J. D. Fletcher, A. Carrington, J. Levallois, M. Nardone, B Vignolle, N. DoironLeyraud, D. LeBoeuf, L. Taillefer, S. Adachi et al., Phys. Rev. Lett. 100, 047004 (2008).

[27] T. Helm et al., unpublished. 